\title{
Accurate Automatic Detection Method to Assist Physicians in Diagnosing Early Nondisplaced Fractures of Femoral Neck
}

\author{
Jin-sheng Fang \\ Minnan Normal University \\ Yen-Yu Chen \\ Minnan Normal University \\ Shang-Lin Hsieh ( $\nabla$ miscyy@seed.net.tw ) \\ China Medical University Hospital \\ Tsung-Li Lin \\ China Medical University Hospital \\ Chih-Yuan Ko \\ China Medical University Hospital
}

\section{Research Article}

Keywords: nondisplaced femoral neck fracture, deep learning network, segmentation

Posted Date: December 17th, 2021

DOI: https://doi.org/10.21203/rs.3.rs-1160814/v1

License: (c) (i) This work is licensed under a Creative Commons Attribution 4.0 International License. Read Full License 


\section{Abstract}

Background - Nondisplaced femoral neck fractures are sometimes misdiagnosed by radiographs. We developed an automatic detection method using deep learning networks to pinpoint femoral neck fractures on radiographs to assist the physicians in making accurate diagnosis in the first place.

Results - A total of approximately 3,840 images of non-displaced Garden type I and II femoral neck fracture cases collected from the Radiology Information System (RIS) from the Picture Archiving and Communication System (PACS) database between 2018 and 2020 from the China Medical University Hospital (CMUH). Two senior orthopedic surgeons from the China Medical University Hospital participated in independently labeling the femoral neck margin and fracture line on these images as the training dataset for the deep learning network. Our proposed accurate automatic detection method, called direction-aware fracture detection network (DAFDNet), consists of two steps, namely region of interest (ROI) segmentation and fracture detection. The first step removes the noise region and pinpoints the femoral neck region. The fracture detection step uses direction-aware deep learning algorithm to mark the exact femoral neck fracture location in the region detected in the first step.

Conclusions - Our proposed DAFDNet demonstrated over $94.8 \%$ accuracy in differentiating non-displaced Garden type I and type II femoral neck fracture cases. Our DAFDNet method outperforms the diagnostic accuracy of general practitioners and orthopedic surgeons in accurately locating Garden type I and type II fractures locations. This study can determine the feasibility of applying artificial intelligence in a clinical setting and how the use of deep learning networks assist physicians in improving the correct diagnosis compared to current traditional orthopedic manual assessments.

\section{Background}

Femoral neck fractures are one of the most common osteoporotic fractures in the elderly and cause substantial morbidity and mortality [1-3]. According to the radiograph-based Garden classification system for assessing fracture severity, femoral neck fractures can be classified into four types, namely nondisplaced Garden I and II and displaced Garden III and IV [4]. Nondisplaced Garden I and II indicate incomplete nondisplaced fractures and complete nondisplaced fractures, respectively. Displaced Garden III and IV are incomplete displaced fractures and complete displaced fractures.

The features of displaced femoral neck fractures are clinically and imaging distinct, whereas those of nondisplaced femoral neck fractures are challenging and receive less attention [5-

8]. Radiographic imaging of nondisplaced femoral neck fractures can be compromised by osteoporosis, obesity, patient position-related reasons, or the use of portable radiographic equipment, and poor image quality, which creates additional difficulties for clinicians [6,9].

Recent advances in artificial intelligence using deep learning techniques, such as deep convolutional neural networks (DCNN), have shown remarkable results for a range of medical tasks as well as for human experts [10-14]. A growing number of studies support that deep learning networks can be trained 
to identify fractures in orthopedic radiographs with satisfactory accuracy [14-16]. Although deep learning has been applied to fracture detection for radiological diagnosis, nondisplaced femoral neck fractures are often overlooked for misdiagnosis, which may result in patients with nondisplaced fractures deteriorating into displaced fractures. Therefore, we propose a new direction-aware fracture detection network, termed as DAFDNet, for automatic detection of femoral neck fractures on anterior-posterior pelvic radiographs. It is well known that Gabor filter is a differentiable band-pass filter with adjustable scales and orientations, and therefore it has been integrated into DCNN [17-19]. Garden type I and Garden type II of femoral neck fractures present different orientations and frequencies in frequency space depending on the patient's imaging location and conditions. By integrating a Gabor filter in the DCNN, the filter is able to fix optimal parameters and help the DCNN learn robust feature presentations. We present this study to validate the accuracy of DCNN in detecting nondisplaced femoral neck fractures, and it shows substantial improvements in performance.

\section{Experiments And Results Dataset and metrics}

The original radiographic images for the experiments, including left or right femoral neck, were acquired from patients and approved by the local Institutional Review Board. We extracted approximately 3,840 radiological images of anterior-posterior views of pelvis from China medical university hospital (CMUH, Taichung, Taiwan) between 2018 and 2020 for nondisplaced ipsilateral femoral neck fracture (Garden type I and II) noted in relevant radiologist reports taken from the Picture Archiving and Communication System database identified through the Radiology Information System. Two senior orthopedic surgeons were involved in the annotation of the images, independently annotating the femoral neck part and the fracture line. In our algorithm, the femoral neck part was used to train the DCNN for ROI segmentation and the fracture line was used to train the DCNN for fracture detection. All labeled images were made under the guidance of professional orthopedist, and new images of $1024 \times 1024$ pixels size were extracted accordingly to reduce the computing time. We used intersection over union (IOU) value between the femoral neck fracture region and the labeled region as assessing metrics, defined as $1 O U=(A \cap B) /(A \cup B)$ where $\cap$ and $\cup$ denote the intersection and union of two sets, $A$ is the intersection of the predicted region and label region and $B$ is the predicted region.

\section{Implementation details}

Fig. 1 shows the femoral neck fracture detection strategy used in this paper, which consists of two phases, namely femoral neck localization and fracture detection. In the first stage, the original image is fed into a segmentation network with matching and alternative methods to accurately localize the femoral neck. In the second stage, a surgeon-made label-trained network is used to localize the exact location of the fracture after the output femoral neck image in the first stage. 
We augment the data by rotating and rescaling the images and labels with various degrees and scales. During training process, 12 images were randomly chosen as input in each training batch. The model was trained by the Adam optimizer with a learning rate initialized to $1 \mathrm{e}^{-5}$ and set to $4 \mathrm{e}^{-5}$ in steps of $1 \mathrm{e}^{-5}$. Two typical DCNNs, i.e., namely U shape Connected convolutional network (U-Net) [21] and Densely Connected convolutional network (DenseNet) [24] were used as the comparing algorithms and their codes were downloaded from GitHub shared by the original authors. The corresponding parameters of these three methods are optimized until the network converges.

\section{Results and comparison}

Fig. 2 shows the pelvic radiographic images with femoral neck fractures and the comparison of the detection results of U-Net, DenseNet and our proposed DAFDNet. As shown in the enlarged view of Figure $2 a$, the fractures are labeled by an experienced surgeon delineated with blue lines. The coordinates of both labeled and predicted results are calculated and plotted in different colors in Figs. $2 b-d$, where the labeled fracture region is plotted in red, while the predicted fracture region results are plotted in yellow. The detection results show that our proposed method is closest to the actual size and area of the label region, while the other methods obtain results with an area several times larger than the labeled region. Therefore, our proposed method gains the largest IOU value among the three methods, which indicates that the fracture detected by our proposed method is the closest to the ground truth.

Fig.3 shows a comparison of the IOU values of our proposed DAFDNet, DenseNet and U-Net. We can see that DAFDNet outperforms the other two methods, and most of the IOU values of DAFDNet are much larger than those of DenseNet and U-Net.

In Table 1, we divided the IOU values into three categories. There are $73.1 \%$ of DAFDNet results above 0.5 (or $50 \%$ ), while none of the other two methods. $21.7 \%$ of the DAFDNet results are between 0.2 and 0.5 (or $[20 \%, 50 \%)$ ), while more than $90 \%$ of DenseNet and U-Net comparison methods have results close to 0.1 (or $[0 \%, 20 \%)$ ). The table also calculates the average IOU values, which are $0.648,0.084$ and 0.062 (or $64.8 \%, 8.4 \%$ and $6.2 \%$ ) for DAFDNet, DenseNet and U-Net, respectively.

Fig. 4 shows the detection results of DAFDNet for different IOU values. the IOU values are divided into three classes, where values above $50 \%$ are shown in (a-c), between $20 \%$ and $50 \%$ are shown in (d-f), and values below $20 \%$ are shown in $(\mathrm{g}-\mathrm{i})$, respectively. In the enlarged view of all sub-images, the red rectangle indicates the labeled region (ground truth) and the yellow rectangle is the fracture region predicted by DAFDNet. It was concluded that DAFDNet achieved better performance than the DenseNet and U-Net comparison methods. The diagnostic correctness of DAFDNet exceeded $94.8 \%$ and the DAFDNet method could assist general practitioners and orthopedic surgeons in the initial diagnosis of Garden type I and II fractures to avoid misclassification and improve the diagnostic correctness.

Table 1 


\begin{tabular}{|llll|}
\hline \multicolumn{1}{|r}{ Methods } & U-Net [21] & DenseNet [24] & DAFDNet \\
IOU(\%) & $(\%)$ & $(\%)$ & $(\%)$ \\
\hline$[50,100]$ & 0 & 0 & 73.1 \\
\hline$[20 \otimes 50)$ & 0.4 & 1.6 & 21.7 \\
\hline$[0,20)$ & 99.6 & 98.4 & 5.2 \\
\hline Average IOU & 6.2 & 8.4 & 64.8 \\
\hline
\end{tabular}

\section{Discussion}

In this study, we propose a new method for detecting femoral neck fractures. The results show that our method is effective in detecting the precise fracture location and outperforms other comparative methods. Our proposed method is implemented in the localization and detection phases, i.e., localization of the femoral neck and detection of fracture detection. The benefit of the localization phase is that by localizing the ROI from the original image, the input data size of DCNN can be greatly reduced. On the one hand, the computation time is saved to a great extent, and on the other hand, the disturbs, such as regions with similar gray distribution in the pelvis image, can be excluded to improve the accuracy of detection. In the fracture detection stage, because the orientation of fracture is random, DAFDNet introduces an orientation-aware algorithm to detect fracture directionality. The use of band-pass filter Gabor enables the network to detect image gray changes by adjusting its frequency or orientation. In addition, attention mechanism and ghost convolution are also involved to improve the performance of DAFDNet.

\section{Conclusion}

Although deep learning has made great advances in medical image processing, few publications have shown clinical utility for detecting femoral neck fractures, especially non-displaced Garden I and Garden II fracture detection. The success of our study in detecting the precise location of nondisplaced fractures provides the first evidence that DCNN can help physicians improve the diagnostic accuracy of nondisplaced Garden I and Garden II fractures. As shown by the predicted rectangular and IOU values of the fractures, our proposed method obtained better results than physician diagnosis. However, fractures were not detected in more than $5.2 \%$ of the images tested, due to the poor contrast of the images. Therefore, better radiographic image quality would greatly improve our approach. 


\section{Materials And Methods \\ DCNN for femoral neck fractures}

Recently, DCNN-based methods have shown great potential efficiency in many areas of medical diagnosis and have encouraged further applied research [20]. The use of DCNN can reduce the need for expensive Computed Tomography (CT) and Magnetic Resonance Imaging (MRI) scans, and its automotive and accurate detection results can reduce the burden on clinicians for urgent identification of fractures [12]. However, the feasibility and efficiency of DCNN in detecting femoral neck fractures remains challenging and has not been fully investigated, needless to say the occult representations of the Garden I and II [14]. To the best of our knowledge, typical DCNN-based medical image segmentation methods [16,21-24], such as U-Net [21] and DenseNet [24], can be applied to detect femoral neck fractures.

These types of fractures may disappear after a series of convolutional operations with the depth of the layers due to the tiny variations in the grayscale distribution in these regions in the radiographic images.

\section{Gabor filter}

Two dimensional Gabor filter is a directional band-pass wavelet filter that is the multiplication of a Gaussian function and a cosine function, defined as follows [17]:

$$
G_{u, v}(\mathrm{z})=\frac{\left\|k_{u, v}\right\|^{2}}{(2 \pi)^{2}} \mathrm{e}^{-\left(\left\|k_{u, v}\right\|^{2}\|\mathrm{z}\|^{2} / 2(2 \pi)^{2}\right)\left[\mathrm{e}^{\mathrm{i} k u, v} \mathrm{z}-\mathrm{e}^{-(2 \pi)^{2} / 2}\right]}
$$

where $k_{u, v}=k_{v} e^{i k_{u}}, k_{v}=(\pi / 2) / \sqrt{2}^{(v-1)}, k_{u}=u \frac{\pi}{U}$ with $v$ and $u$ are the frequency

and orientation, respectively. Substantially, the Gabor transform is a windowed short-time Fourier transform that is able to extract features locally or of certain frequency components. The direction and frequency selection properties make the Gabor filter sensitive to certain types of boundaries.

In radiography, the orientations of nondisplaced femoral neck fracture is related to the patient's position, where the frequency components lie in certain ranges. Therefore, in our study, a multiple-direction Gabor filter with adjusted frequency bands was engaged as a input layer to the DCNN to detect the tiny changes in the grayscale distribution in Garden type I and type II.

\section{Attention mechanism}

The attention mechanism was invented to tell DCNN where or what features to focus on, which was demonstrated to significantly improve the effectiveness of model performance $[25,26]$. Squeeze-andExcitation attention network (SENet) exploits the squeeze function and the excitation function, i.e., the global average pooling operation and the Sigmoid function, respectively, to encode inter-channel 
information [27]. This simple and innovative model provides a significant performance improvement for DCNN, but ignores the location information that is important for capturing features [25]. Therefore, several extension studies such as Bottle-Attention-Module (BAM) [28], Convolutional Block-AttentionModule (CBAM) [29], and Spatial and Channel-wise Attention (SCA) [30] have been proposed to further extract spatial and channel information and improve the network effectiveness. Self-attention DCNN models Attention in Attention network ( $A^{2} \mathrm{Net}$ ) divides the attention branches into attention and nonattention branches to maximize the use of high-contributing information and minimize the suppression of redundant information [31]. Although $\mathrm{A}^{2} \mathrm{Net}$ exhibits excellent performance, the large amount of computation requires significant hardware facility costs. In summary, this study uses the SCA strategy to obtain spatial and channel-wise information.

\section{Direction-aware segmentation network}

In this section, we introduce the implementation details of the proposed DAFDNet model, including the attention mechanism Ghost convolution and the details of the model architecture.

\section{Squeeze-and-Excitation Ghost convolution}

GhostNet was first proposed in reference [32] to reduce the computation consumption by replacing the ordinary convolution with a simple linear transformation. A Ghost module divides the result of convolution into two parts. The first part involves ordinary convolution, while the other part uses a series of linear transformations to generate more feature maps, as shown in Fig.5a. By using this strategy, the lightweight Ghost module produces more feature maps with inexpensive operations and performs better than other lightweight DCNNs, which also accelerates the learning process. However, the linear transformation does not focus on cross-channel relationships, which have proven to be robust in object detection. Therefore, each Squeeze-and-Excitation (SE) block consisting of a global average pooling and two fully connected layers is embedded in the Ghost module instead of linear transformation, as show in Fig. 5b. The weights calculated from the SE blocks are then multiplied with the input convolution results by channel and concatenated with the original convolution results to generate the final feature maps.

\section{Model architecture}

As shown in Fig.6, DAFDNet uses the popular encoder-decoder framework to first encode the input image by focusing on the attention Ghost convolutional module and Gabor-filter convolution. The input image is manipulated with the Ghost module, and then two down-sampling operations are performed to gain global feature maps with different resolutions, i.e., $G h o M_{1}, G h o M_{2}$, and $G h o M_{3}$, respectively. The output feature map $G h o M_{i}$ in the Ghost module is represented as follows 


$$
G h o M_{i}=\operatorname{Ghost} \operatorname{Conv}\left(G_{i}, L_{i}\right), i=1,2, \ldots \mathrm{n}
$$

\section{where $L_{i}$ is the ith input feature map and $G_{i}$ is the ith Ghost module.}

In the process of bypassing the Gabor convolution, we use an 8-direction Gabor with diverse scales to extract the boundary of the femoral neck fracture, and then perform two down-sampling operations to generate two additional batches of shrinking feature maps. Then, we obtain the feature maps $G a b_{i}$ from the Gabor convolution, which is represented as follows

$\operatorname{Gab}_{i}=\operatorname{GaborConv}\left(G F_{i}(\theta, s), L_{i}\right) i=1,2, \ldots \mathrm{n}$

where $G F_{i}(\theta, s)$ is the Gabor-filter with directions $\theta$ and scales $s$. Afterwards, in our study, $G a b_{1}, G a b_{2}$ and $G b_{3}$ are concatenated with $G h o M_{1}, G h o M_{2}$ and $G h o M_{3}$, respectively, to get aggregated feature maps $G G_{1}, G G_{2}$ and $G G_{3}$ expressed by

$G G_{i}=\operatorname{Concat}\left(G a b_{i}, G h o M_{i}\right) \quad i=1,2, \ldots \mathrm{n}$

Then, the extracted features are refined with a $2 \times 2$ average pooling layer, a $3 \times 3$ convolution layer and a Batch Normalization layer to obtain the $P F_{j}$ feature map. We concatenate the corresponding $G h o M_{i}$ and $P F_{i}$ with the same resolution to obtain $A_{j}$ where the results are processed with the attention module, respectively. The feature maps with lower resolution, such as $A_{3}, A_{2}$ as shown in Fig.6, are resized with a $2 \times 2$ up-sampling layer before being concatenated to the feature maps with higher resolution. In general, the process can be expressed as follows

$$
\left\{\begin{array}{rl}
\text { Concat }_{i+1} & =\text { Concat }\left(\text { Upsampling }\left(A_{i+2}\right), A_{i+1}\right) \\
\text { Concat }_{i} & =\operatorname{Concat}\left(\text { Upsampling }\left(A_{i+1}\right), A_{i}\right)
\end{array} i=1,2, \ldots, n\right.
$$

Hence, the output of our network is result of the sequential operations of expressions (2-5) with a $2 \times 2$ upsampling layer to recover the feature size as the input image and followed by a $1 \times 1$ convolution layer to reduce the dimension of channels.

The loss function of DAFDNet is mean square error that can be expressed as

$$
L(\vartheta)=\frac{1}{N} \sum_{i=1}^{N}\left\|\operatorname{DASNet}\left(I_{i}^{I N}\right)-I_{i}^{G T}\right\|_{2}
$$

where $\vartheta$ represents the learnable parameter of DAFDNet and $\|.\|_{2}$ is $\mathrm{L}_{2}$-norm. $I_{i}^{I N}$,

$I_{i}^{G T}$ denotes the input images and the corresponding ground truth, respectively. 


\section{Abbreviations}

RIS: Radiology Information System

PACS: Picture Archiving and Communication System

CMUH: China Medical University Hospital

DAFDNet: direction-aware fracture detection network

ROI: region of interest

DCNN: deep convolutional neural network

IOU: intersection over union

U-Net: U shape Connected convolutional network

DenseNet: Densely Connected convolutional network

CT: Computed Tomography

MRI: Magnetic Resonance Imaging

SENet: Squeeze-and-Excitation network

BAM: Bottle-Attention-Module

CBAM: Convolutional Block-Attention-Module

SCA: Spatial and Channel-wise Attention

A2Net: Attention in Attention network

\section{Declarations}

- Ethics approval and consent to participate:

Not applicable

\section{- Consent for publication:}

Not applicable

- Competing interests: 
The authors declare that they have no competing interests

\section{- Funding:}

in writing the manuscript

1. The Education Department of Fujian Province, China

2. Xiamen University, China

3. China Medical University Hospital, Taiwan

\section{- Authors' contributions:}

Fang Jin-Sheng analyzed the data disease and tested the DCNN program. Chen Yen-Yu performed the examination of the results. Hsieh Shang-Lin was a major contributor in writing the manuscript. Lin Tsung$\mathrm{Li}$ and Ko Chih-Yuan labeled the femoral neck margin and fracture line on these images as the training dataset for the deep learning network. All authors have read and approved the final manuscript.

\section{- Acknowledgements:}

The authors would like to thank Fujian Provincial Natural Science Foundation Projects (2021 J011005), Department of Education of Fujian Province (JAT190361), Xiamen University (20191201) and China Medical University Hospital for financially supporting this research.

\section{- Availability of data and material:}

The datasets used or analyzed during the current study are available from the corresponding author on reasonable request.

\section{References}

1. Papadimitriou N, Tsilidis K, Orfanos P, Benetou V, et al. Burden of hip fracture using disabilityadjusted life-years: a pooled analysis of prospective cohorts in the CHANCES consortium. Lancet Public Health. 2017 May;2(5): e239-e246. doi: 10.1016/S2468-2667(17)30046-4. Epub 2017 Apr 11. PMID: 29253489.

2. Brauer $C A$, Coca-Perraillon $M$, Cutler $D M$, Rosen $A B$. Incidence and mortality of hip fractures in the United States. JAMA, 2009; 302:1573-1579.

3. Marks R. Hip fracture epidemiological trends, outcomes, and risk factors, 1970-2009. Int J Gen Med, 2010; 3:1-17. 
4. Garden RS. Low-angle fixation in fractures of the femoral neck. J Bone Joint Surg Br. 1961; 43-B: 647-663.

5. Anthony V. Florschutz, MD, PhD, Joshua R. Langford, MD, George J. Haidukewych, MD, and Kenneth J. Koval, MD. Femoral Neck Fractures: Current Management. J Orthop Trauma, Volume 29, Number 3, March 2015

6. Hoskins W, Rayner J, Sheehy R, Claireaux H, Bingham R, Santos R, et al. The effect of patient, fracture and surgery on outcomes of high energy neck of femur fractures in patients aged 15-50. Hip Int. 2019; 29(1):77-82.

7. Gjertsen J, Fevang J, Matre K, et al. Clinical outcome after undisplaced femoral neck fractures[J]. Acta Orthopaedica, 2011, 82(3):268.

8. Mutasa S, Varada S, Goel A, et al. Advanced Deep Learning Techniques Applied to Automated Femoral Neck Fracture Detection and Classification[J]. Journal of Digital Imaging, 2020, 33(4).

9. Kim KC, Ha YC, Kim TY, Choi JA, Koo KH. Initially missed occult fractures of the proximal femur in elderly patients: implications for need of operation and their morbidity, Arch Orthop Trauma Surg, 2010; 130: 915-20.

10. Prevedello LM, Erdal BS, Ryu JL et al. Automated critical test findings identification and online notification system using artificial intelligence in imaging. Radiology. 2017; 285:923-931.

11. Arif Al SMMR, Knapp K, Slabaugh G. Fully automatic cervical vertebrae segmentation framework for X-ray images. Comput Methods Prog Biomed. 2018; 157:95-111.

12. Gale W, Oakden-Rayner L, Carneiro G, et al. Detecting hip fractures with radiologist-level performance using deep neural networks. 2017.

13. Kazi A, Albarqouni S, Sanchez A J, et al. Automatic Classification of Proximal Femur Fractures Based on Attention Models. International Workshop on Machine Learning in Medical Imaging, 2017.

14. Cheng C, Ho TY, Lee TY, Chang CC, Chou CC, Chen CC, et al. Application of a deep learning algorithm for detection and visualization of hip fractures on plain pelvic radiographs. Eur Radiol. 2019; 29:5469-77.

15. Olczak J, Fahlberg N, Maki A, Razavian AS, Jilert A, Stark A, et al. Artificial intelligence for analyzing orthopedic trauma radiographs: deep learning algorithms-are they on par with humans for diagnosing fractures?. Acta Orthop. 2017; 88:581-6.

16. Cheng $\mathrm{C}$, Chen $\mathrm{CC}$, Cheng FJ, Chen HW, Su YS, Yeh CN, et al. A human algorithm integration system for hip fracture detection on plain radiography: system development and validation study. JMIR Med Informatics. 2020; 8:1-13

17. S. Luan, C. Chen, B. Zhang, J. Han and J. Liu. Gabor Convolutional Networks. IEEE Transactions on Image Processing, vol. 27, no. 9, pp. 4357-4366, Sept. 2018, doi: 10.1109/TIP.2018.2835143.

18. Yao H, Li C, Dan H, et al. Gabor Feature Based Convolutional Neural Network for Object Recognition in Natural Scene. International Conference on Information Science \& Control Engineering. IEEE, 2016. 
19. Sarwar S S, Panda P, Roy K. Gabor Filter Assisted Energy Efficient Fast Learning Convolutional Neural Networks. IEEE, 2017.

20. Kermany D S, Goldbaum M, Ca I W, et al. Identifying Medical Diagnoses and Treatable Diseases by Image-Based Deep Learning. Cell, 2018, 172(5):1122-1131.e9.

21. Ronneberger O, Fischer P, Brox T. U-Net: Convolutional Networks for Biomedical Image Segmentation. International Conference on Medical Image Computing and Computer-Assisted Intervention, 2015.

22. Simonyan K, Zisserman A. Very Deep Convolutional Networks for Large-Scale Image Recognition. arXiv, 2014.

23. He K, Zhang, Ren S, et al. Deep Residual Learning for Image Recognition. IEEE, 2016.

24. Huang G, Liu Z, Laurens V, et al. Densely Connected Convolutional Networks. IEEE Computer Society, 2016.

25. Hou Q, Zhou D, Feng J. Coordinate Attention for Efficient Mobile Network Design. 2021.

26. Oktay O, Schlemper J, Folgoc L, et al. Attention U-Net: Learning Where to Look for the Pancreas. 2018.

27. Jie H, Li S, Gang S, et al. Squeeze-and-Excitation Networks. IEEE Transactions on Pattern Analysis and Machine Intelligence, 2017, PP (99).

28. Park J, Woo S, Lee J Y, et al. BAM: Bottleneck Attention Module. 2018.

29. Woo S, Park J, Lee J Y, et al. CBAM: Convolutional Block Attention Module. European Conference on Computer Vision. Springer, Cham, 2018.

30. Long C, Zhang H, Xiao J, et al. SCA-CNN: Spatial and Channel-Wise Attention in Convolutional Networks for Image Captioning. 2017 IEEE Conference on Computer Vision and Pattern Recognition (CVPR). IEEE, 2016.

31. Chen H, Gu J, Zhang Z. Attention in Attention Network for Image Super-Resolution. 2021.

32. Han K, Wang Y, Tian Q, et al. GhostNet: More Features From Cheap Operations. 2020 IEEE/CVF Conference on Computer Vision and Pattern Recognition (CVPR). IEEE, 2020.

\section{Figures}




\section{Phase One: Femoral Neck Location}

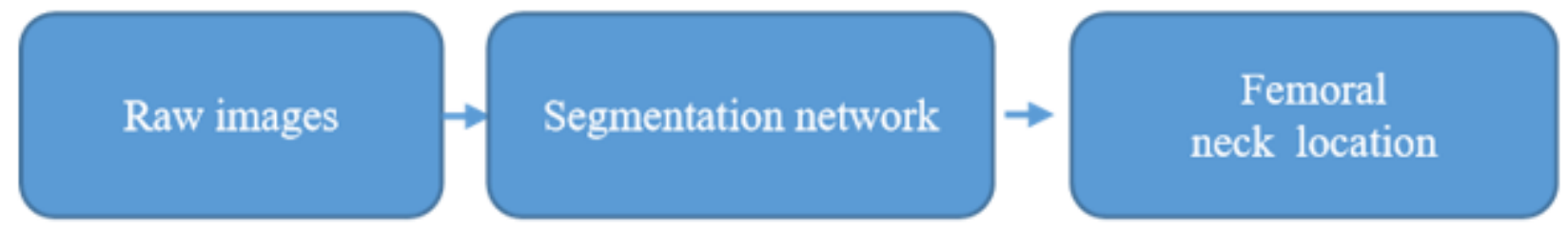

\section{Phase Two: Fracture Detection}

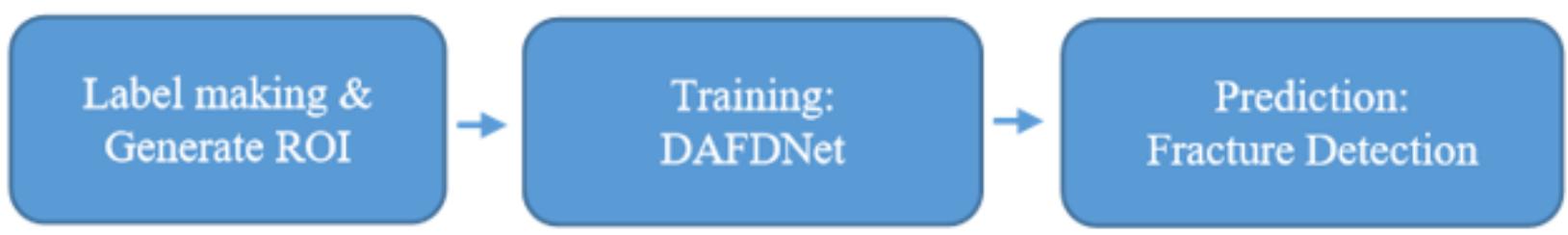

Figure 1

Workflow of the fracture detection strategy. Two phases are included, femoral neck localization and fracture detection.
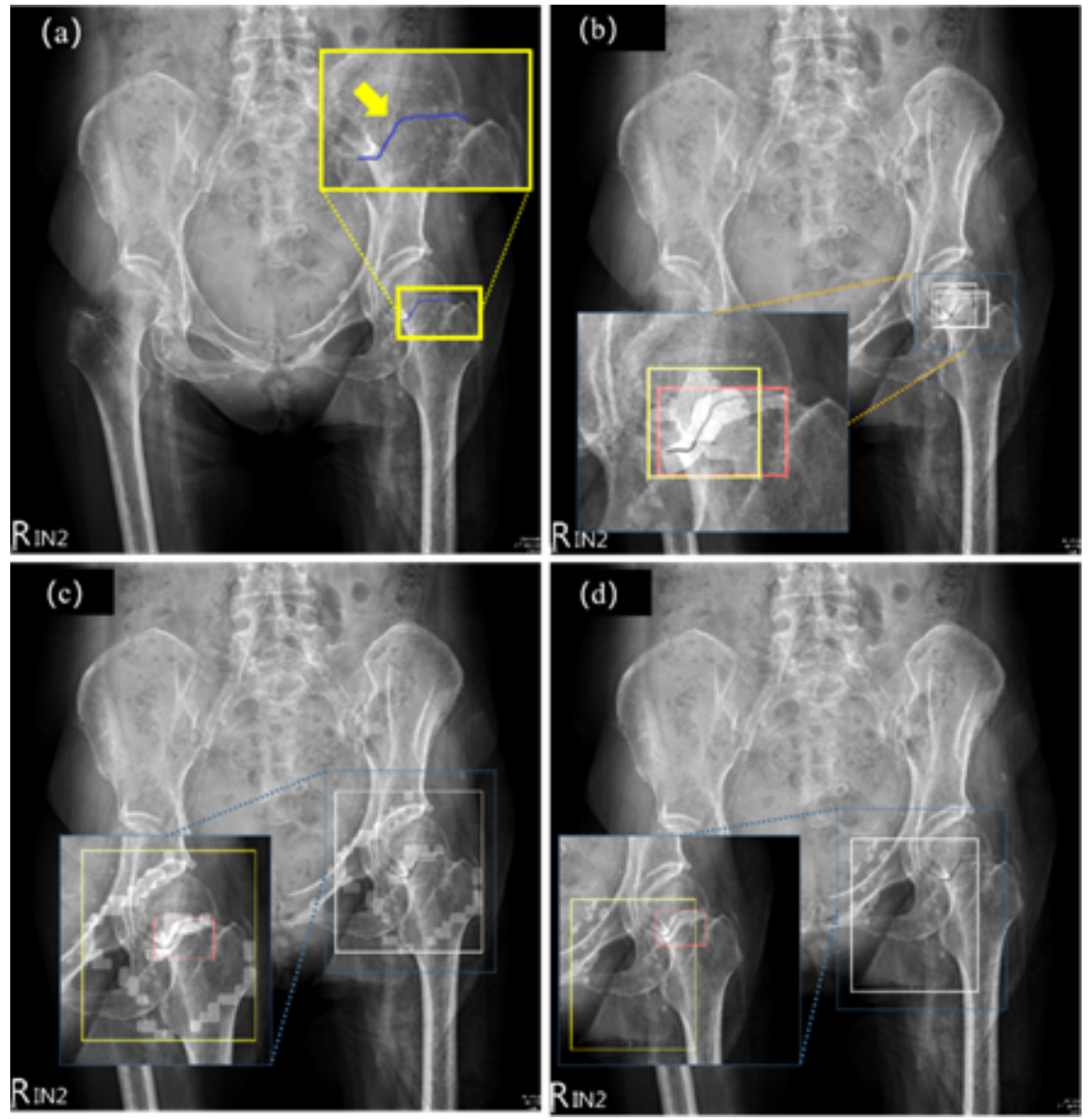
Figure 2

Radiographic images of pelvis and femoral neck fractures and the detection results of U-Net, DenseNet and our proposed DAFDNet. (a) Imaging of the pelvis with femoral neck fracture labeled by blue line in the magnified view. (b) Fracture detected by DAFDNet, (c) fracture detected by U-Net and (d) fracture location detected by DenseNet. As shown in the enlarged view, the fracture detection range for each method is in the yellow rectangle and the physician-delineated labels are in the red rectangle.

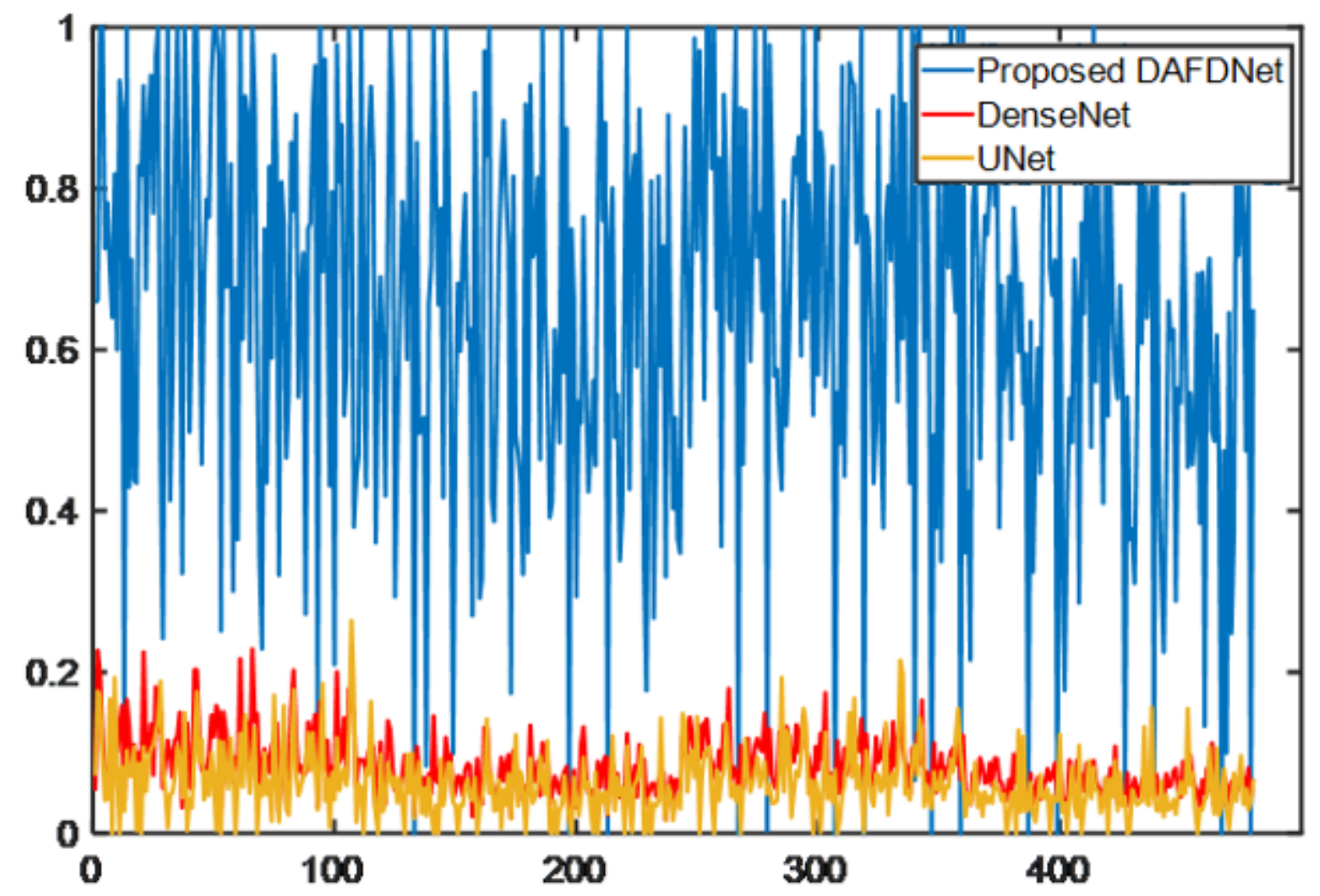

Figure 3

Comparison of IOU values of all the test images predicted by the three methods. Most of the IOU values of DAFDNet are larger than those of DenseNet and U-Net. 


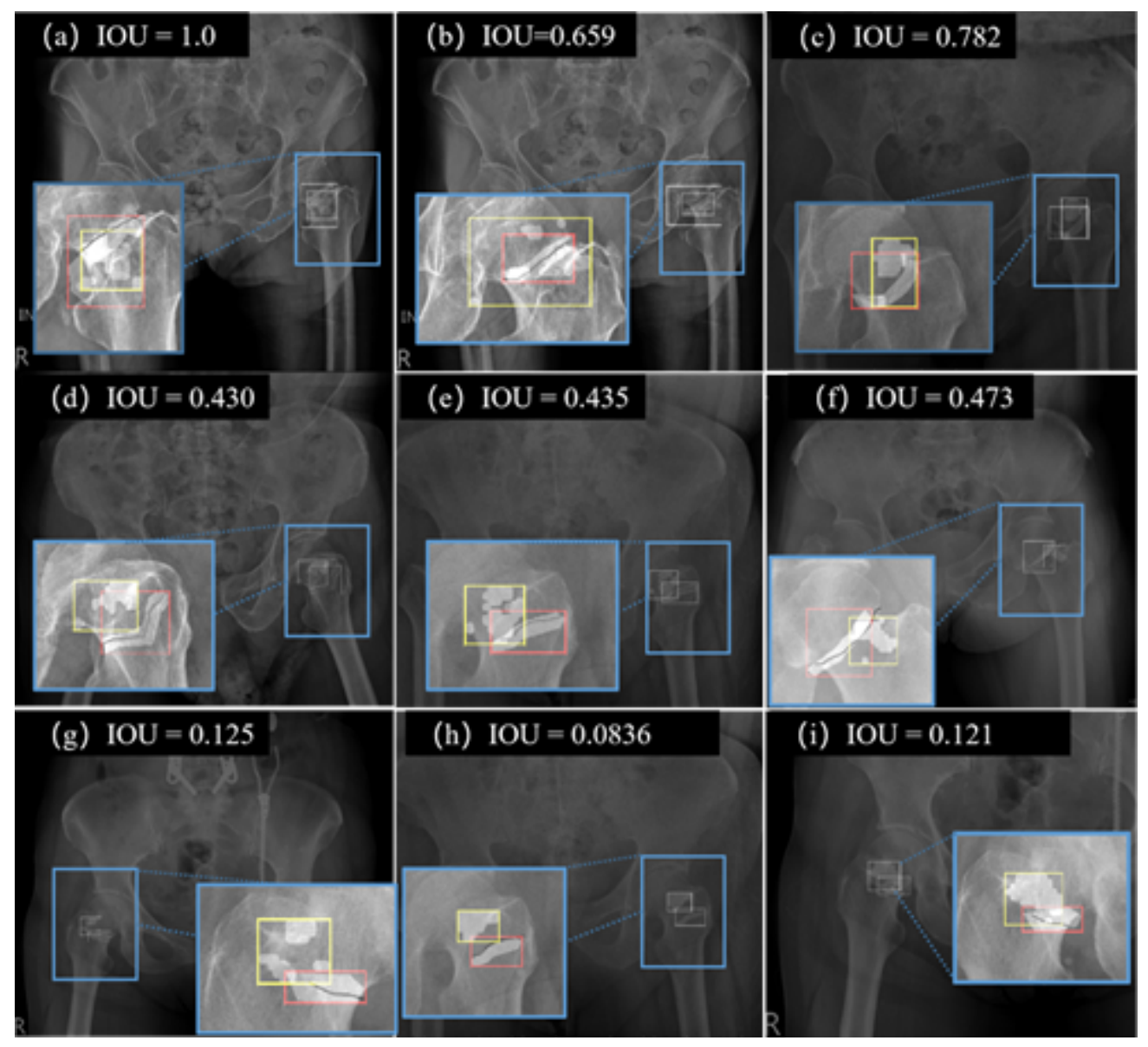

\section{Figure 4}

Detection results of DAFDNet for different IOU values.

(a)

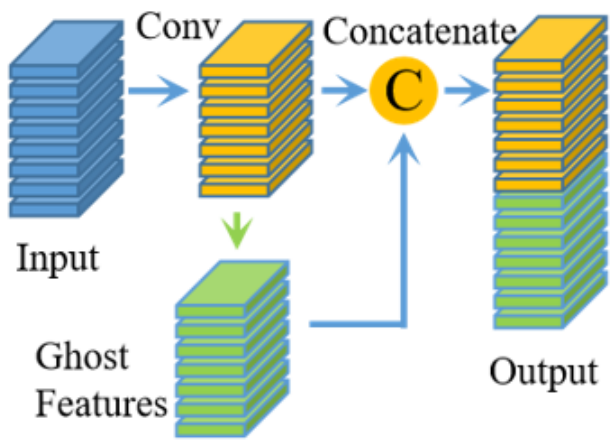

(b)

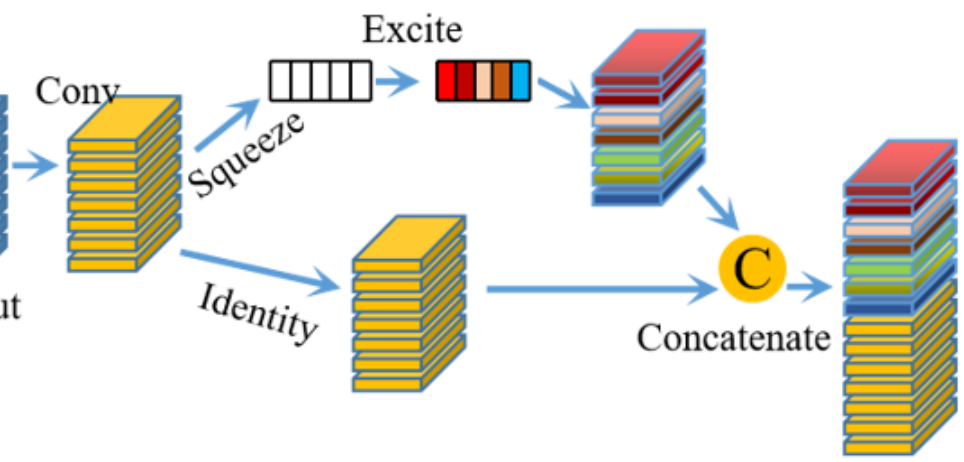

\section{Figure 5}

Original Ghost convolution and SE Ghost module. (a) Ghost convolution, which use a simple linear transformation to generate more features; (b) SE Ghost module, which incorporates the SE attention mechanism into the Ghost module to discriminate the weight of each channel. 


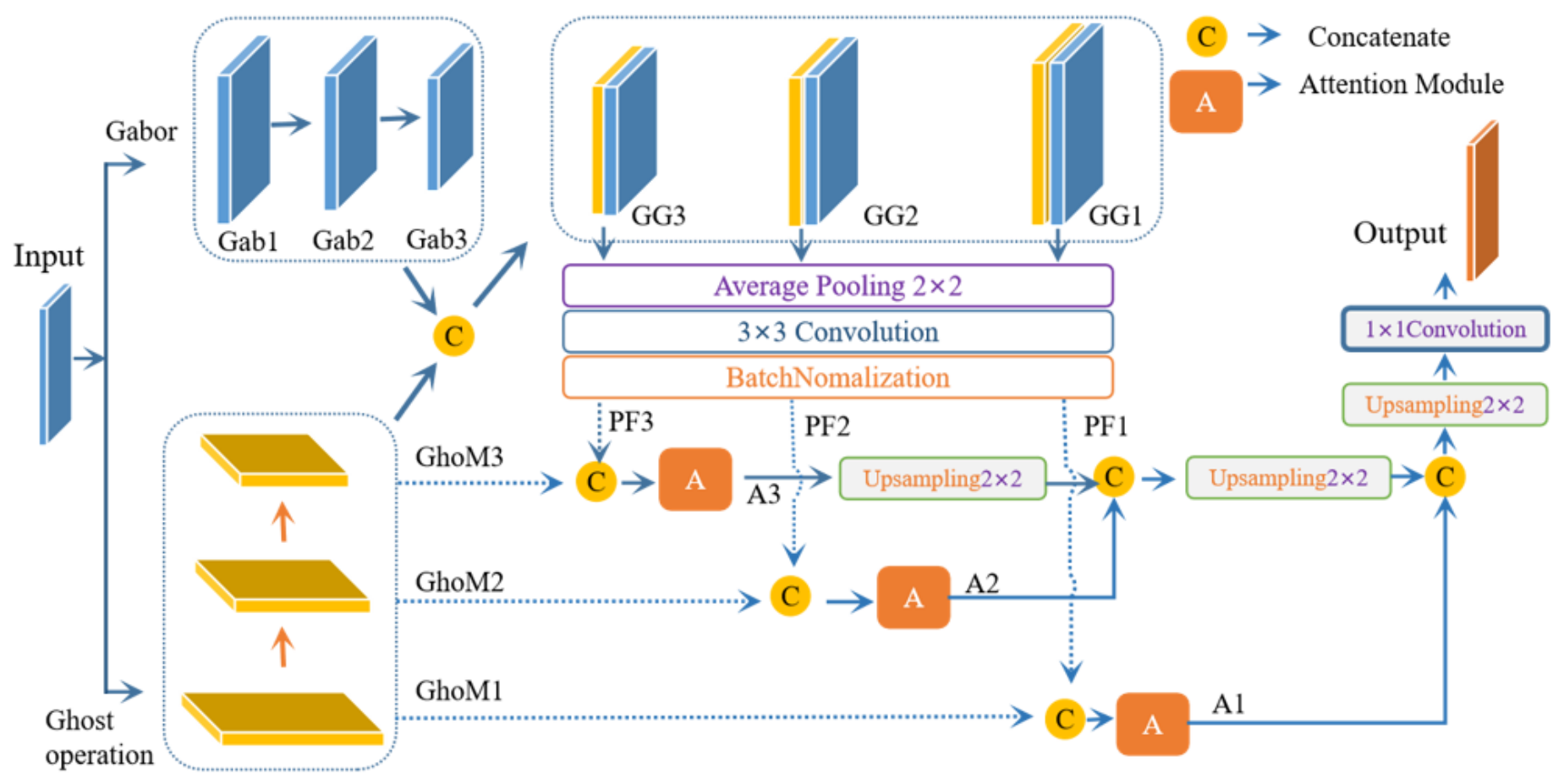

Figure 6

Schematic diagram of the proposed DAFDNet. 